\title{
Green Functions for Sub-Laplacian on Half Spaces of the Heisenberg Group
}

\author{
$\mathrm{Na} \mathrm{Wei}^{\mathrm{a}}$, Pengcheng Niu ${ }^{\mathrm{a}}$, Jialin Wang ${ }^{\mathrm{b}}$ \\ ${ }^{a}$ Department of Applied Mathematics; Key Laboratory of Space Applied Physics and Chemistry, Ministry of \\ Northwestern Polytechnical University Education Xi'an, Shaanxi Province, 710129, China \\ ${ }^{b}$ Key Laboratory of Numerical Simulation Technology of Jiangxi Province Gannan Normal University \\ Ganzhou, Jiangxi Province, 341000, China
}

\begin{abstract}
Green functions for sub-Laplacian on the domains in the Heisenberg group are derived, which can be used to solve partial differential equations subject to specific initial conditions or boundary conditions. Then the integral formulas for sub-Laplace equation on characteristic and non-characteristic half spaces are given, respectively.
\end{abstract}

Index Terms: Heisenberg group; half space; Green function

(C) 2011 Published by MECS Publisher. Selection and/or peer review under responsibility of the Research Association of Modern Education and Computer Science.

\section{Introduction}

Green functions play an important role in the study of partial differential equations. Itô [1] studied the Green type kernels on the half space in $\square^{n}$. Wong [2] gave a formula of the Green function of a degenerate elliptic operator on $\square^{2}$ related to Heisenberg group. The aim of this paper is to discuss the Green functions for sub-Laplacian on non-characteristic and characteristic half spaces of Heisenberg group, and deduce the integral formulas for the Dirichlet problems of the sub-Laplace equations.

We recall some notations and known results about the Heisenberg group $H^{n}$. The group $H^{n}$ is a two step nilponent Lie group $\left(\square^{2 n+1}, \circ\right)$ with group law given by

$$
\xi \circ \tilde{\xi}=(x, y, t) \circ(\tilde{x}, \tilde{y}, \tilde{t})=(x+\tilde{x}, y+\tilde{y}, t+\tilde{t}+2(\tilde{x} \cdot y-x \cdot \tilde{y}))
$$

in $\square^{2 n+1}$, where $\xi=(z, t)=(x, y, t), \quad \tilde{\xi}=(\tilde{z}, \tilde{t})=(\tilde{x}, \tilde{y}, \tilde{t})$

$\in \square^{2 n+1}, x \cdot y$ denote the usual inner product in $\square^{n}$.

The sub-Laplace operator in $H^{n}$ is

* Corresponding author.

E-mail address: Pengchengniu @ nwpu.edu.cn 


$$
\Delta_{H^{n}}=\sum_{i=1}^{n}\left(X_{i}^{2}+Y_{i}^{2}\right),
$$

where $X_{i}=\partial / \partial x_{i}+2 y_{i} \cdot \partial / \partial t, Y_{i}=\partial / \partial y_{i}-2 x_{i} \cdot \partial / \partial t \quad($ for $i=1, \ldots, n), T=\partial / \partial t$. The generalized gradient on $H^{n}$ is $\nabla_{H^{n}}=\left(X_{1}, \cdots, X_{n}, Y_{1}, \cdots, Y_{n}\right) . H^{n}$ allows to a family of dilations $\left\{\delta_{\lambda}: \lambda>0\right\}$ defined by $\delta_{\lambda}(\xi)=\left(\lambda z, \lambda^{2} t\right), \lambda>0$. The Jacobi determinant of $\delta_{\lambda}$ is $\lambda^{Q}$, where $Q=2 n+2$ is the homogeneous dimension of $H^{n}$.

It is well known that the fundamental solution of sub-Laplace operator $-\Delta_{H^{n}}$ at the origin is

$$
\Gamma(\xi)=c_{Q} / d(\xi)^{Q-2},
$$

where $c_{Q}>0$ is a suitable constant, $d(\xi)=\left(|z|^{4}+t^{2}\right)^{1 / 4}$

(see [3]). If $\tilde{\xi} \in H^{n}$, then $\tilde{\xi}^{-1}=-\tilde{\xi}$ by the group law. Let $d(\xi, \tilde{\xi})=d\left(\tilde{\xi}^{-1} \circ \xi\right)$ denote the distance between $\xi$ and $\tilde{\xi}$ in $H^{n}$ (see [4]). By translation and (1), the fundamental solution of $-\Delta_{H^{n}}$ with pole at $\tilde{\xi}$ can be written as

$$
\Gamma(\xi, \tilde{\xi})=\Gamma\left(\tilde{\xi}^{-1} \circ \xi\right)=c_{Q} / d\left(\tilde{\xi}^{-1} \circ \xi\right)^{Q-2} .
$$

It is easy to know that

$$
\nabla \Gamma(\xi, \tilde{\xi})=-(Q-2) c_{Q} d\left(\tilde{\xi}^{-1} \circ \xi\right)^{1-Q} \cdot \nabla d\left(\tilde{\xi}^{-1} \circ \xi\right) .
$$

In general, we let $\Omega_{r}=\left\{\xi \in H^{n} \mid d(\xi)<r\right\}$ and $\partial \Omega_{r}=\left\{\xi \in H^{n} \mid d(\xi)=r\right\}$ denote the Heisenberg ball and sphere centered at the origin with radius $r$, respectively.

Note that $H^{n}$ is a non-abelian Lie group, whose stratification such that there exist characteristic points on the boundary of the smooth domain of $H^{n}$. Thus it is difficult for the Dirichlet problem of sub-Laplace equation to have good regularity and we have to introduce non- characteristic domain in the following. Let $\Phi(z, t)$ be the boundary function in $\Omega \subset H^{n}$. If $\left|\nabla_{H^{n}} \Phi(z, t)\right| \neq 0$, then $\Omega$ is called the non-characteristic domain in $H^{n}$. If there exist some points on the boundary such that $\left|\nabla_{H^{n}} \Phi(z, t)\right|=0$, then $\Omega$ is called the characteristic domain and these points are named characteristic points. In this paper, by simple calculation we know that

$$
\Pi_{1}=\left\{\xi=\left(x_{1}, \cdots, x_{n}, y_{1}, \cdots, y_{n}, t\right) \in H^{n} \mid x_{1}>0\right\}
$$

(by taking $\Phi=x_{1}$ ) and

$$
\Pi_{0}=\left(\xi=\left(x_{1}, \cdots, x_{n}, y_{1}, \cdots, y_{n}, t\right) \in H^{n} \mid t>0\right)
$$

(by taking $\Phi=t$ ) are non-characteristic half space and characteristic half space in $H^{n}$, respectively.

The paper is organized as follows: In Section II, we give the Green function of sub-Laplacian on the general domain of $H^{n}$. In Section III and Section IV, we give the Green functions of sub-Laplacian on the non-characteristic half space and characteristic half space of $H^{n}$ by the results in Section II, respectively. We also show the integral formulas for solutions of the Dirichlet problem of sub-Laplacian. By the discussion in this paper, we can see that the Green functions in these two half spaces are different in nature.

\section{Green function on general domain}


By [5], one has $\Delta_{H^{n}}=\operatorname{div}(A \nabla)$, where

$$
A=\left(\begin{array}{ccc}
I_{n} & 0 & 2 y \\
0 & I_{n} & -2 x \\
2 y & -2 x & 4|z|^{2}
\end{array}\right)
$$

and $I_{n}$ denotes the identity matrix in $\square^{n}$. The usual gradient in $\square^{2 n+1}$ is denoted by $\nabla=(\partial / \partial x, \partial / \partial y, \partial / \partial t)^{T}$.

Let $\Omega \subset H^{n}$ be a domain with smooth boundary. By [5], for $v \in C^{\infty}\left(H^{n}\right)$,

$\int_{\Omega}\left(u \Delta_{H^{n}} v-v \Delta_{H^{n}} u\right) d \xi=\int_{\Omega \Omega}(u A \nabla v \cdot \vec{n}-v A \nabla u \cdot \vec{n}) d H_{2 n},(4)$ where $\vec{n}$ denotes the unit outward normal vector of $\partial \Omega, d H_{2 n}$ denotes the $2 n$-dimensional Hausdorff measure in $\square^{2 n+1}$. Assume that $u \in C^{\infty}(\bar{\Omega})$ and let $\Omega_{r}(\eta) \subset \Omega$ be the Heisenberg ball centered at $\eta$ with radius $r$. Letting $v \equiv 1$ in (4), we have

$$
\int_{\Omega} \Delta_{H^{n}} u d \xi=\int_{\partial \Omega} A \nabla u \cdot \vec{n} d H_{2 n} .
$$

We substitute $v$ with $\Gamma\left(\xi^{\prime-1} \circ \xi\right)$ in $\Omega \backslash \Omega_{r}$,

$\int_{\Omega \mid \Omega_{r}} \Gamma \Delta_{H^{n}} u d \xi=\Gamma \int_{\partial \Omega} A \nabla u \cdot \vec{n} d H_{2 n}+\Gamma \int_{\partial \Omega_{r}} A \nabla u \cdot \vec{n} d H_{2 n} \quad-\int_{\partial \Omega} u A \nabla \Gamma \cdot \vec{n} d H_{2 n}-\int_{\partial \Omega_{r}} u A \nabla \Gamma \cdot \vec{n} d H_{2 n} \cdot \quad$ ( 6

Note that $\vec{n}=\nabla d /|\nabla d|$ on $\partial \Omega$ and $\vec{n}=-\nabla d /|\nabla d|$ on $\partial \Omega_{r}$. By (2.25), (2.26) in [5], we get

and

$$
\lim _{r \rightarrow 0^{+}} \int_{\partial \Omega_{r}} u A \nabla \Gamma \cdot \vec{n} d H_{2 n}=-u(\eta)
$$

$$
\lim _{r \rightarrow 0^{+}} \Gamma \int_{\partial \Omega_{r}} A \nabla u \cdot \vec{n} d H_{2 n}=0 .
$$

Thus, let $r \rightarrow 0^{+}$in (6) and then

$$
u(\eta)=\int_{\partial \Omega}(u A \nabla \Gamma \cdot \vec{n}-\Gamma A \nabla u \cdot \vec{n}) d H_{2 n}
$$

$+\int_{\Omega} \Gamma \Delta_{H^{n}} u d \xi \eta \in \Omega$.

In particular, if $\Delta_{H^{n}} u=0$ in $\Omega$, then we have the following basic integration formulas,

$$
u(\eta)=\int_{\partial \Omega}(u A \nabla \Gamma \cdot \vec{n}-\Gamma A \nabla u \cdot \vec{n}) d H_{2 n}
$$

Let $h(\xi, \eta)$ belong to $C^{\infty}(\bar{\Omega})$ with respect to $\xi$ and satisfies $-\Delta_{H^{n}} h(\xi)=0$ in $\Omega$. For $u(\xi) \in C^{\infty}(\bar{\Omega})$, we take $v=h$ in (4), then

$$
\int_{\partial \Omega}(u A \nabla h \cdot \vec{n}-h A \nabla u \cdot \vec{n}) d H_{2 n}+\int_{\Omega} h \Delta_{H^{n}} u d \xi=0 .
$$

Let us add (8) and (9) and set

$$
G(\xi, \eta)=\Gamma\left(\eta^{-1} \circ \xi\right)+h(\xi, \eta)
$$

and $G=0$ on $\partial \Omega$, then $h(\xi, \eta)=-\Gamma\left(\eta^{-1} \circ \xi\right)$, so

$$
u(\eta)=\int_{\partial \Omega} u(\xi) A \nabla G \cdot \vec{n} d H_{2 n} .
$$

We say that $G=G(\xi, \eta)$ is the Green function on $\Omega$.

\section{Green function on non-characteristic half space}


We consider the Dirichlet problem of sub - Laplace equation on the non-characteristic half space $\Pi_{1}$ in $H^{n}$ :

$$
\left\{\begin{array}{c}
-\Delta_{H^{n}} u(\xi)=0, \quad \xi \in \Pi_{1} ; \\
u(\xi)=\varphi\left(\xi^{\prime}, t\right), \quad \xi \in\left\{x_{1}=0\right\} ; \\
\lim _{\|\xi\| \rightarrow \infty} u(\xi)=0,
\end{array}\right.
$$

where $\xi=(x, y, t)=\left(x_{1}, \cdots, x_{n}, y_{1}, \cdots, y_{n}, t\right)=\left(x_{1}, \xi^{\prime}, t\right)$, $\xi^{\prime}=\left(x_{2}, \cdots, x_{n}, y_{1}, \cdots, y_{n}\right)$.

Let $\xi, \eta \in \Pi_{1} \quad$ it is easy to know that the symmetric point of $\eta=(\hat{x}, \hat{y}, \hat{t})=\left(\hat{x}_{1}, \hat{x}_{2}, \cdots, \hat{x}_{n}, \hat{y}_{1}, \cdots, \hat{y}_{n}, t\right)=\left(\hat{x}_{1}, \eta^{\prime}, \hat{t}\right)$

is $\eta^{*}=\left(-\hat{x}_{1}, \eta^{\prime}, \hat{t}\right)$. Selecting $h(\xi, \eta)=-\Gamma\left(\left(\eta^{*}\right)^{-1} \circ \xi\right)$ in (10), the Green function on half space is

$$
G(\xi, \eta)=\Gamma\left(\eta^{-1} \circ \xi\right)-\Gamma\left(\left(\eta^{*}\right)^{-1} \circ \xi\right) \text {. }
$$

Because of $\eta^{*} \notin \Pi_{1}, h(\xi, \eta)$ satisfies $-\Delta_{H^{n}} h=0$ with respect to $\xi \in \Pi_{1}$. When $\xi$ is over $\Pi_{1}$, we have

$$
G(\xi, \eta)=\Gamma\left(\eta^{-1} \circ \xi\right)-\Gamma\left(\left(\eta^{*}\right)^{-1} \circ \xi\right)=0 .
$$

Therefore, by (10), for any $\eta \in \Pi_{1}, \eta=\left(\hat{x}_{1}, \eta^{\prime}, \hat{t}\right), \hat{x}_{1}>0$, it follows

$$
u(\eta)=\int_{x_{1}=0} \varphi\left(\xi^{\prime}, t\right) A \nabla G \cdot \vec{n} d H_{2 n}^{\prime} .
$$

Next, we will calculate $A \nabla G \cdot \vec{n}$. To do this, we note that for $\xi, \eta \in \Pi_{1}$,

$$
\begin{aligned}
d(\xi, \eta)= & d\left(\eta^{-1} \circ \xi\right) \\
=\{ & \left\{\left(x_{1}-\hat{x}_{1}\right)^{2}+\sum_{i=2}^{n}\left(x_{i}-\hat{x}_{i}\right)^{2}+\sum_{i=1}^{n}\left(y_{i}-\hat{y}_{i}\right)\right\}^{2} \\
& \left.+\left(t-\hat{t}+2 \sum_{i=1}^{n} \hat{x}_{i} y_{i}-2 \sum_{i=1}^{n} x_{i} \hat{y}_{i}\right)^{2}\right\}^{1 /} ;
\end{aligned}
$$

$$
\begin{aligned}
& \left.\frac{\partial d(\xi, \eta)}{\partial x_{1}}\right|_{x_{1}=0}=1 / 4 d(\xi, \eta)^{-3}\left\{2 \left[\left(x_{1}-\hat{x}_{1}\right)^{2}\right.\right. \\
& \left.+\sum_{i=2}^{n}\left(x_{i}-\hat{x}_{i}\right)^{2}+\sum_{i=1}^{n}\left(y_{i}-\hat{y}_{i}\right)^{2}\right] \cdot\left(2 x_{1}-2 \hat{x}_{1}\right)+ \\
& \left.2\left(t-\hat{t}+2 \sum_{i=1}^{n} \hat{x}_{i} y_{i}-2 \sum_{i=1}^{n} x_{i} \hat{y}_{i}\right)\left(-2 \hat{y}_{1}\right)\right\}\left.\right|_{x_{1}=0} \\
& =-d^{\prime}(\xi, \eta)^{-3}\left\{\left[\hat{x}_{1}^{2}+\sum_{i=2}^{n}\left(x_{i}-\hat{x}_{i}\right)^{2}+\sum_{i=1}^{n}\left(y_{i}-\hat{y}_{i}\right)^{2}\right] \cdot \hat{x}_{1}\right. \\
& \left.+\left(t-\hat{t}+2 \sum_{i=1}^{n} \hat{x}_{i} y_{i}-2 \sum_{i=2}^{n} x_{i} \hat{y}_{i}\right) \hat{y}_{1}\right\} \\
& \square-M_{1} / d^{\prime}(\xi, \eta)^{3} ; \\
& \left.\frac{\partial d(\xi, \eta)}{\partial t}\right|_{x_{1}=0}=\left.\frac{1}{4 d(\xi, \eta)^{3}} \cdot 2\left(t-\hat{t}+2 \sum_{i=1}^{n} \hat{x}_{i} y_{i}-2 \sum_{i=1}^{n} x_{i} \hat{y}_{i}\right)\right|_{x_{1}=0} \\
& =1 / 2 d^{\prime}(\xi, \eta)^{-3}\left(t-\hat{t}+2 \sum_{i=1}^{n} \hat{x}_{i} y_{i}-2 \sum_{i=2}^{n} x_{i} \hat{y}_{i}\right) \\
& \square M_{2} / d^{\prime}(\xi, \eta)^{3} ;
\end{aligned}
$$

$d\left(\xi, \eta^{*}\right)=d\left(\left(\eta^{*}\right)^{-1} \circ \xi\right)$ 


$$
\begin{aligned}
& =\left\{\left[\left(x_{1}+\hat{x}_{1}\right)^{2}+\sum_{i=2}^{n}\left(x_{i}-\hat{x}_{i}\right)^{2}+\sum_{i=1}^{n}\left(y_{i}-\hat{y}_{i}\right)^{2}\right]^{2}\right. \\
& \left.+\left(t-\hat{t}-2 \hat{x}_{1} y_{1}+2 \sum_{i=2}^{n} \hat{x}_{i} y_{i}-2 \sum_{i=1}^{n} x_{i} \hat{y}_{i}\right)^{2}\right\}^{1 / 4},\left.\frac{\partial d\left(\xi, \eta^{*}\right)}{\partial x_{1}}\right|_{x_{1}=0}=\frac{1}{4 d\left(\xi, \eta^{*}\right)^{3}}\left\{2 \left[\left(x_{1}+\hat{x}_{1}\right)^{2}+\right.\right. \\
& \left.\sum_{i=2}^{n}\left(x_{i}-\hat{x}_{i}\right)^{2}+\sum_{i=1}^{n}\left(y_{i}-\hat{y}_{i}\right)^{2}\right] \cdot\left(2 x_{1}+2 \hat{x}_{1}\right)+ \\
& \left.2 t-\hat{t}-2 \hat{x}_{1} y_{1}+2 \sum_{i=2}^{n} \hat{x}_{i} y_{i}-2 \sum_{i=1}^{n} x_{i} \hat{y}_{i}\left(-2 \hat{y}_{1}\right)\right\}\left.\right|_{x_{1}=0} \\
& =d^{\prime}\left(\xi, \eta^{*}\right)^{-3}\left\{\left[\hat{x}_{1}^{2}+\sum_{i=2}^{n}\left(x_{i}-\hat{x}_{i}\right)^{2}+\sum_{i=1}^{n}\left(y_{i}-\hat{y}_{i}\right)^{2}\right] \cdot \hat{x}_{1}-\left(t-\hat{t}-2 \hat{x}_{1} y_{1}+2 \sum_{i=2}^{n} \hat{x}_{i} y_{i}-2 \sum_{i=2}^{n} x_{i} \hat{y}_{i}\right) \hat{y}_{1}\right\} \\
& \square N_{1} / d^{\prime}\left(\xi, \eta^{*}\right)^{3} ; \\
& \left.\left(\partial d\left(\xi, \eta^{*}\right) / \partial t\right)\right|_{x_{1}=0} \\
& =1 /\left.4 d\left(\xi, \eta^{*}\right)^{-3} \cdot 2\left(t-\hat{t}-2 \hat{x}_{1} y_{1}+2 \sum_{i=2}^{n} \hat{x}_{i} y_{i}-2 \sum_{i=1}^{n} x_{i} \hat{y}_{i}\right)\right|_{x_{1}=0} \\
& =1 / 2 d^{\prime}\left(\xi, \eta^{*}\right)^{-3}\left(t-\hat{t}-2 \hat{x}_{1} y_{1}+2 \sum_{i=2}^{n} \hat{x}_{i} y_{i}-2 \sum_{i=2}^{n} x_{i} \hat{y}_{i}\right) \square N_{2} / d^{\prime}\left(\xi, \eta^{*}\right)^{3},
\end{aligned}
$$$$
\text { where } M_{1}=\left\{\left[\hat{x}_{1}^{2}+\sum_{i=2}^{n}\left(x_{i}-\hat{x}_{i}\right)^{2}+\sum_{i=1}^{n}\left(y_{i}-\hat{y}_{i}\right)^{2}\right] \cdot \hat{x}_{1}\right.
$$$$
\left.+\left(t-\hat{t}+2 \sum_{i=1}^{n} \hat{x}_{i} y_{i}-2 \sum_{i=2}^{n} x_{i} \hat{y}_{i}\right) \hat{y}_{1}\right\}
$$$$
N_{1}=\left[\hat{x}_{1}^{2}+\sum_{i=2}^{n}\left(x_{i}-\hat{x}_{i}\right)^{2}+\sum_{i=1}^{n}\left(y_{i}-\hat{y}_{i}\right)^{2}\right] \cdot \hat{x}_{1}
$$$$
-\left(t-\hat{t}-2 \hat{x}_{1} y_{1}+2 \sum_{i=2}^{n} \hat{x}_{i} y_{i}-2 \sum_{i=2}^{n} x_{i} \hat{y}_{i}\right) \hat{y}_{1}
$$$$
M_{2}=1 / 2\left(t-\hat{t}+2 \sum_{i=1}^{n} \hat{x}_{i} y_{i}-2 \sum_{i=2}^{n} x_{i} \hat{y}_{i}\right)
$$$$
N_{2}=1 / 2\left(t-\hat{t}-2 \hat{x}_{1} y_{1}+2 \sum_{i=2}^{n} \hat{x}_{i} y_{i}-2 \sum_{i=2}^{n} x_{i} \hat{y}_{i}\right)
$$

$d^{\prime}(\xi, \eta)$ and $d^{\prime}\left(\xi, \eta^{*}\right)$ denotes $d(\xi, \eta)$ and $d\left(\xi, \eta^{*}\right)$ at $x_{1}=0$, respectively.

By the expression $A \nabla d=\left(\frac{\partial d}{\partial x}+2 y \frac{\partial d}{\partial t}, \frac{\partial d}{\partial y}-2 x \frac{\partial d}{\partial t}, 2 y \frac{\partial d}{\partial x}-2 x \frac{\partial d}{\partial y}+4|z|^{2} \frac{\partial d}{\partial t}\right)^{T}$ and (3), we get $\left.(A \nabla G) \cdot \vec{n}\right|_{x_{1}=0}=\left.\left[A \nabla \Gamma(\xi, \eta)-A \nabla \Gamma\left(\xi, \eta^{*}\right)\right] \cdot \vec{n}\right|_{x_{1}=0}=-(Q-2) c_{Q}\left(d^{\prime}(\xi, \eta)^{1-Q} A \nabla d(\xi, \eta)\right.$ $\left.-d^{\prime}\left(\xi, \eta^{*}\right)^{Q} A \nabla d \xi \ddot{\eta}\right) n_{n_{1}=0}$

$$
\begin{aligned}
& =-\left.(Q-2) c_{Q}\left[d^{\prime}(\xi, \eta)^{1-Q}\left(\frac{\partial d(\xi, \eta)}{\partial x_{1}}+2 y_{1} \frac{\partial d(\xi, \eta)}{\partial t}\right)-d^{\prime}\left(\xi, \eta^{*}\right)^{1-Q}\left(\frac{\partial d\left(\xi, \eta^{*}\right)}{\partial x_{1}}+2 y_{1} \frac{\partial d\left(\xi, \eta^{*}\right)}{\partial t}\right)\right]\right|_{x_{1}=0} \\
& =-(Q-2) c_{Q}\left[d^{\prime}(\xi, \eta)^{1-Q}\left(\frac{-M_{1}}{d^{\prime}(\xi, \eta)^{3}}+2 y_{1} \frac{M_{2}}{d^{\prime}(\xi, \eta)^{3}}\right)-d^{\prime}\left(\xi, \eta^{*}\right)^{1-Q}\left(\frac{N_{1}}{d^{\prime}\left(\xi, \eta^{*}\right)^{3}}+2 y_{1} \frac{N_{2}}{d^{\prime}\left(\xi, \eta^{*}\right)^{3}}\right)\right]
\end{aligned}
$$




$$
\begin{aligned}
=(Q-2) c_{Q}\left[d^{\prime}(\xi, \eta)^{-2-Q}\left(M_{1}-2 y_{1} M_{2}\right)\right. \\
\left.+d^{\prime}\left(\xi, \eta^{*}\right)^{-2-Q}\left(N_{1}+2 y_{1} N_{2}\right)\right] .
\end{aligned}
$$

Substituting it into (13), yields

Theorem 1. For any $\eta \in \Pi_{1}$, the integral formula of solutions for Dirichlet problem (12) is

$u(\eta)=(Q-2) c_{Q} \cdot \int_{x_{1}=0} \varphi\left(\xi^{\prime}, t\right)\left[d^{\prime}(\xi, \eta)^{-2-Q}\left(M_{1}-2 y_{1} M_{2}\right)+d^{\prime}\left(\xi, \eta^{*}\right)^{-2-Q}\left(N_{1}+2 y_{1} N_{2}\right)\right] d \xi^{\prime} d t$, where $M_{1}, M_{2}, N_{1}, N_{2} \quad$ are defined as above.

\section{Green function on characteristic half space}

Consider the Dirichlet problem on $\Pi_{0}$ in $H^{n}$ :

$$
\left\{\begin{array}{c}
-\Delta_{H^{n}} u(\xi)=0, \quad \xi \in \Pi_{0} ; \\
u(\xi)=\varphi\left(\xi^{\prime}\right), \quad \xi \in\{t=0\} ; \\
\lim _{\|\xi\| \rightarrow \infty} u(\xi)=0,
\end{array}\right.
$$

where $\xi=\left(x_{1}, \cdots, x_{n}, y_{1}, \cdots, y_{n}, t\right)=\left(\xi^{\prime}, t\right)$. The symmetric point of $\eta=(\hat{x}, \hat{y}, \hat{t})=\left(\eta^{\prime}, \hat{t}\right) \in \Pi_{0}$ is $\eta^{*}=\left(\eta^{\prime},-\hat{t}\right)$. Let us take $h(\xi, \eta)=-\Gamma\left(\left(\eta^{*}\right)^{-1} \circ \xi\right)$ in (10), the Green function on half space is given by

$$
G(\xi, \eta)=\Gamma\left(\eta^{-1} \circ \xi\right)-\Gamma\left(\left(\eta^{*}\right)^{-1} \circ \xi\right) .
$$

Because $\eta^{*} \notin \Pi_{0}, h(\xi, \eta)$ is sub-harmonic with respect to $\xi \in \Pi_{0}$. When $\xi$ is over the hyper-plane $t=0$, we have

$$
G(\xi, \eta)=\Gamma\left(\eta^{-1} \circ \xi\right)-\Gamma\left(\left(\eta^{*}\right)^{-1} \circ \xi\right)=0 .
$$

Hence, by (10), for any $\eta=\left(\eta^{\prime}, \hat{t}\right) \in \Pi_{0}, \hat{t}>0$, we get

$$
u(\eta)=\int_{t=0} \varphi\left(\xi^{\prime}\right) A \nabla G \cdot \vec{n} d H_{2 n}^{\prime} .
$$

We will calculate $A \nabla G \cdot \vec{n}$. For $\xi, \eta \in \Pi_{0}$, we have

$$
\begin{aligned}
& d(\xi, \eta)=d\left(\eta^{-1} \circ \xi\right)= \\
& \left\{\left[\sum_{i=1}^{n}\left(x_{i}-\hat{x}_{i}\right)^{2}+\sum_{i=1}^{n}\left(y_{i}-\hat{y}_{i}\right)^{2}\right]^{2}+\left(t-\hat{t}+2 \sum_{i=1}^{n} \hat{x}_{i} y_{i}-2 \sum_{i=1}^{n} x_{i} \hat{y}_{i}\right)^{2}\right\}^{1 / 4}, \partial d(\xi, \eta) / \partial x_{i}= \\
& 1 /\left.4 d(\xi, \eta)^{-3}\left\{2 \sum_{i=1}^{n}\left(x_{i}-\hat{x}_{i}\right)^{2}+\sum_{i=1}^{n}\left(y_{i}-\hat{y}_{i}\right)^{2} \cdot 2\left(x_{i}-\hat{x}_{i}\right)\right\}\right|_{t=0} \\
& =d^{\prime}(\xi, \eta)^{-3}\left\{\left[\sum_{i=1}^{n}\left(x_{i}-\hat{x}_{i}\right)^{2}+\sum_{i=1}^{n}\left(y_{i}-\hat{y}_{i}\right)^{2}\right] \cdot\left(x_{i}-\hat{x}_{i}\right)\right\} \square P\left(x_{i}-\hat{x}_{i}\right) / d^{\prime}(\xi, \eta)^{3}, i=1, \cdots, n ; \\
& \partial d(\xi, \eta) / \partial y_{i}= \\
& \left.\frac{1}{4 d(\xi, \eta)^{3}}\left\{2\left[\sum_{i=1}^{n}\left(x_{i}-\hat{x}_{i}\right)^{2}+\sum_{i=1}^{n}\left(y_{i}-\hat{y}_{i}\right)^{2}\right] \cdot 2\left(y_{i}-\hat{y}_{i}\right)\right\}\right|_{t=0} \\
& =d^{\prime}(\xi, \eta)^{-3}\left\{\left[\sum_{i=1}^{n}\left(x_{i}-\hat{x}_{i}\right)^{2}+\sum_{i=1}^{n}\left(y_{i}-\hat{y}_{i}\right)^{2}\right] \cdot\left(y_{i}-\hat{y}_{i}\right)\right\} \square P\left(y_{i}-\hat{y}_{i}\right) / d^{\prime}(\xi, \eta)^{3}, \quad i=1, \cdots, n ; \\
& \left.\frac{\partial d(\xi, \eta)}{\partial t}\right|_{t=0}=\left.\frac{1}{4 d(\xi, \eta)^{3}}\left\{2\left(t-\hat{t}+2 \sum_{i=1}^{n} \hat{x}_{i} y_{i}-2 \sum_{i=1}^{n} x_{i} \hat{y}_{i}\right)\right\}\right|_{t=0}
\end{aligned}
$$




$$
\begin{aligned}
& =1 / 2 d^{\prime}(\xi, \eta)^{-3}\left(-\hat{t}+2 \sum_{i=1}^{n} \hat{x}_{i} y_{i}-2 \sum_{i=1}^{n} x_{i} \hat{y}_{i}\right) \\
& \square M^{\prime} / d^{\prime}(\xi, \eta)^{3} ; \\
& d\left(\xi, \eta^{*}\right)=d\left(\left(\eta^{*}\right)^{-1} \circ \xi\right)= \\
& \left.-d^{\prime}\left(\xi, \eta^{*}\right)^{1-Q} A \nabla d\left(\xi, \eta^{*}\right)\right]\left.\cdot \vec{n}\right|_{t=0} \\
& =-(Q-2) c_{Q}\left\{d^{\prime}(\xi, \eta)^{1-Q}\right. \text {. } \\
& \left.\left[\sum_{i=1}^{n}\left(2 y_{i} \frac{\partial d(\xi, \eta)}{\partial x_{i}}-2 x_{i} \frac{\partial d(\xi, \eta)}{\partial y_{i}}\right)+4|z|^{2} \frac{\partial d(\xi, \eta)}{\partial t}\right]\right\}\left.\right|_{t=0} \\
& \left\{\left[\sum_{i=1}^{n}\left(x_{i}-\hat{x}_{i}\right)^{2}+\sum_{i=1}^{n}\left(y_{i}-\hat{y}_{i}\right)^{2}\right]^{2}\right. \\
& \left.+\left(t+\hat{t}+2 \sum_{i=1}^{n} \hat{x}_{i} y_{i}-2 \sum_{i=1}^{n} x_{i} \hat{y}_{i}\right)^{2}\right\}^{1 / 4} .
\end{aligned}
$$

Therefore

$\partial d\left(\xi, \eta^{*}\right) / \partial x_{i}=$

$$
\begin{aligned}
& \left.\frac{1}{4 d\left(\xi, \eta^{*}\right)^{3}}\left\{2\left[\sum_{i=1}^{n}\left(x_{i}-\hat{x}_{i}\right)^{2}+\sum_{i=1}^{n}\left(y_{i}-\hat{y}_{i}\right)^{2}\right] \cdot 2\left(x_{i}-\hat{x}_{i}\right)\right\}\right|_{t=0}=d^{\prime}\left(\xi, \eta^{*}\right)^{-3}\left\{\left[\sum_{i=1}^{n}\left(x_{i}-\hat{x}_{i}\right)^{2}+\sum_{i=1}^{n}\left(y_{i}-\hat{y}_{i}\right)^{2}\right] \cdot\left(y_{i}-\hat{y}_{i}\right)\right\} \\
& \square P\left(x_{i}-\hat{x}_{i}\right) / d^{\prime}\left(\xi, \eta^{*}\right)^{3}, \quad i=1, \cdots, n ; \\
& \partial d\left(\xi, \eta^{*}\right) / \partial y_{i}= \\
& \left.\frac{1}{4 d\left(\xi, \eta^{*}\right)^{3}}\left\{2\left[\sum_{i=1}^{n}\left(\left(x_{i}-\hat{x}_{i}\right)^{2}+\left(y_{i}-\hat{y}_{i}\right)^{2}\right)\right] \cdot 2\left(y_{i}-\hat{y}_{i}\right)\right\}\right|_{t=0} \\
& =d^{\prime}\left(\xi, \eta^{*}\right)^{-3}\left\{\left[\sum_{i=1}^{n}\left(x_{i}-\hat{x}_{i}\right)^{2}+\sum_{i=1}^{n}\left(y_{i}-\hat{y}_{i}\right)^{2}\right] \cdot\left(y_{i}-\hat{y}_{i}\right)\right\} \\
& \square P\left(y_{i}-\hat{y}_{i}\right) / d^{\prime}\left(\xi, \eta^{*}\right)^{3}, \quad i=1, \cdots, n ; \\
& \left.\frac{\partial d\left(\xi, \eta^{*}\right)}{\partial t}\right|_{t=0}=\left.\frac{1}{4 d\left(\xi, \eta^{*}\right)^{3}}\left\{2\left(t+\hat{t}+2 \sum_{i=1}^{n} \hat{x}_{i} y_{i}-2 \sum_{i=1}^{n} x_{i} \hat{y}_{i}\right)\right\}\right|_{t=0}=1 / 2 d^{\prime}\left(\xi, \eta^{*}\right)^{-3}\left(\hat{t}+2 \sum_{i=1}^{n} \hat{x}_{i} y_{i}-2 \sum_{i=1}^{n} x_{i} \hat{y}_{i}\right) \\
& \square N^{\prime} / d^{\prime}\left(\xi, \eta^{*}\right)^{3} .
\end{aligned}
$$

where $M^{\prime}=1 / 2\left(-\hat{t}+2 \sum_{i=1}^{n} \hat{x}_{i} y_{i}-2 \sum_{i=2}^{n} x_{i} \hat{y}_{i}\right)$,

$$
\begin{aligned}
& N^{\prime}=1 / 2\left(\hat{t}+2 \sum_{i=1}^{n}\left(\hat{x}_{i} y_{i}-x_{i} \hat{y}_{i}\right)\right), \\
& P=\sum_{i=1}^{n}\left(\left(x_{i}-\hat{x}_{i}\right)^{2}+\left(y_{i}-\hat{y}_{i}\right)^{2}\right) ;
\end{aligned}
$$

$d^{\prime}(\xi, \eta), d^{\prime}\left(\xi, \eta^{*}\right)$ denotes $d(\xi, \eta), d\left(\xi, \eta^{*}\right)$ at $t=0$,

respectively. By (3),

$\left.(A \nabla G) \cdot \vec{n}\right|_{t=0}=\left.\left[A \nabla \Gamma(\xi, \eta)-A \nabla \Gamma\left(\xi, \eta^{*}\right)\right] \cdot \vec{n}\right|_{t=0}$

$=-(Q-2) c_{Q}\left[d^{\prime}(\xi, \eta)^{1-Q} A \nabla d(\xi, \eta)\right.$ 


$$
\begin{aligned}
& +(Q-2) c_{Q}\left\{d ^ { \prime } ( \xi , \eta ^ { * } ) ^ { 1 - Q } \cdot \left[\sum_{i=1}^{n}\left(2 y_{i} \frac{\partial d\left(\xi, \eta^{*}\right)}{\partial x_{i}}-2 x_{i} \frac{\partial d\left(\xi, \eta^{*}\right)}{\partial y_{i}}\right)\right.\right. \\
& \left.+\left.4\left|z^{2}\right| \frac{\partial d\left(\xi \eta^{*}\right)}{\partial t}\right|_{t}\right\}_{0} \\
& =-(Q-2) c_{Q}\left[d^{\prime}(\xi, \eta)^{-2-Q} \cdot\left(P \sum_{i=1}^{n}\left(2 x_{i} \hat{y}_{i}-2 y_{i} \hat{x}_{i}\right)+4|z|^{2} M^{\prime}\right)\right]+ \\
& (Q-2) c_{Q}\left[d^{\prime}\left(\xi, \eta^{*}\right)^{-2-Q} \cdot\left(P \sum_{i=1}^{n}\left(2 x_{i} \hat{y}_{i}-2 y_{i} \hat{x}_{i}\right)+4|z|^{2} N^{\prime}\right)\right] .
\end{aligned}
$$

Using (14), we have

Theorem 2. For any $\eta \in \Pi_{0}$, the integral formula for Dirichlet problem (14) on $\Pi_{0}$ is $u(\eta)=-(Q-2) c_{Q}$.

$\int_{t=0} \varphi\left(\xi^{\prime}\right)\left(\frac{\left(P \sum_{i=1}^{n}\left(2 x_{i} \hat{y}_{i}-2 y_{i} \hat{x}_{i}\right)+4|z|^{2} M^{\prime}\right)}{d^{\prime}(\xi, \eta)^{2+Q}}-\frac{\left(P \sum_{i=1}^{n}\left(2 x_{i} \hat{y}_{i}-2 y_{i} \hat{x}_{i}\right)+4|z|^{2} N^{\prime}\right)}{d^{\prime}\left(\xi, \eta^{*}\right)^{2+Q}}\right) d \xi^{\prime}, \quad$ where $M^{\prime}, N^{\prime}, P$ are defined as above.

\section{Conclusion}

We obtain several kinds of Green functions in different domains of the Heisenberg group, which extend some results in the Euclidean space to the more general case. Theses results are new and very important in the study of partial differential equations.

\section{References}

[1] M. Itô, "On the Green type kernels on the half space in $\square^{n} "$ ". Ann. Inst. Fourier Grenoble, vol. 28, no. 2, pp. 85-105, 1978.

[2] M. -W. Wong, "Weyl transforms, the heat kernel and Green function of a degenerate elliptic operator", Ann. Global Anal. Geom., no. 28, pp. 271-283, 2005

[3] G. B. Folland, "A fundamental solution for a subelliptic operator", Bull. Amer. Math. Soc., no. 79, pp. 373-376, 1973.

[4] J. Cygan, "Wiener's test for the Brownian motion on the Heisenberg group", Colloquium Math., no. 39, pp. 367-373, 1978.

[5] N . Garofalo, E. Lanconelli, "Frequency functions on the Heisenberg group, the uncertainty principle and unique continuation", Ann. Inst. Fourier Grenoble, no. 40, pp. 313-356, 1990. 